\title{
THE ROLE OF CONVECTION IN REDUCING \\ NONADIABATICITY AND MODE COUPLING IN \\ CEPHEIDS
}

\author{
J. ZALEWSKI* \\ Astronomical Institute, Tóhoku Lniversity, Aoba-ku, Sendai 980. Japan
}

\begin{abstract}
The effect of convection on the strength of coupling is examined. It is found that in Cepheid models the inclusion of convection smooths the sharp peak of entropy perturbation in the ionization region and reduces significantly the coupling.
\end{abstract}

\section{Introduction}

The nonadiabatic amplitude equations formalism has been extensively studied in the case of Cepheids and other stars populating the instability strip (see e.g. Buchler, Moskalik and Kovács, 1991). In radiative models, for which the formalism was developed (Buchler and Goupil, 1984, hereinafter BG) it is found that perturbations of thermodynamic quantities in the ionization zones become very large and numerical problems appear in the calculation of coupling coefficients (Pesnell and Buchler, 1986). Buchler and Kovács (1987, hereinafter BK, see also Kovács and Buchler, 1989) have devised a method in which the coupling coefficients are derived from a fit to the results of nonlinear calculations, however in this approach one has to verify the applicability of amplitude equations by comparing their prediction with the behavior of nonlinear models. To avoid the difficulties of the BG formalism Takeuti, Yamakawa and Ishida (1991, hereinafter TYI) have introduced a modified nonadiabatic formalism for amplitude equations.

With TYI formalism it is found that in radiative Cepheid models the coupling is very strong - the nonadiabatic coupling coefficients are by about two orders of magnitude larger than those calculated from adiabatic eigenfunctions. Basing on these results it has been suggested by Tanaka et al. (1991) that the inclusion of convection may reduce the strength of coupling.

Here I describe the effect the convection has on linear pulsations and on the coupling coefficients. The convection in the background models was described by the mixing length theory, and in linear pulsation calculations the usual approximation viz. that $\nabla \cdot F_{c}^{\prime}=0$ (see Unno et al., 1989) has been made.

* On leave from N. Copernicus Astronomical Center, Warsaw, Poland. 


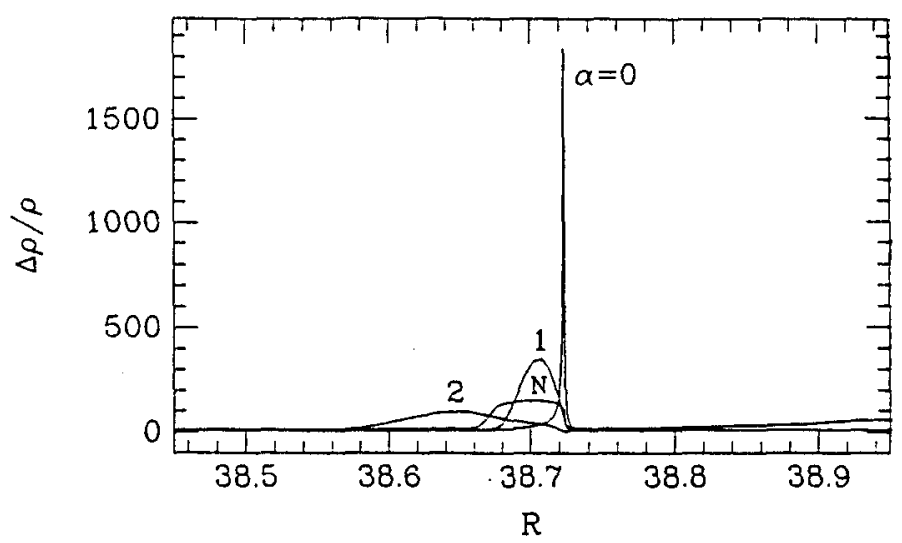

Fig. 1. Dependence of $|\Delta \rho / \rho|$ on $\alpha$ in a Cepheid model. Curves are labeled with $\alpha$. The curve labeled $N$ represents the Lagrangean density fluctuation at the instant of maximum compression in the ionization region (see text).

\section{The Effect of Convection on Linear Eigenvectors}

In purely radiative models of Cepheids the amplitudes of density or temperature eigenfunctions (calculated from linear nonadiabatic equations) are sharply peaked in the ionization region of $\mathrm{H} / \mathrm{He}$ (see e.g. Stellingwerf, 1990), partly due to strong nonlinearity of opacity and partly due to sharp gradients occurring in this region which are due to the lack of effective energy transport mechanism in these models.

To examine the effect of convection series of static models parametrized by $\alpha=l / H_{p}$ (see Paczynski, 1969), the ratio of mixing length to the pressure scale height, have been computed. The linear nonadiabatic pulsations have been calculated using Dziembowshi's (1977) code. The dependence of the density eigenfunction on is shown in Fig. 1. The temperature perturbation behaves in a similar way. As it is seen the amplitude and steepness of the peak are strongly reduced relative to purely radiative model due to the decreased radiation flux. This also leads to the decrease of nonadiabaticity in the ionization zones because the total pressure perturbation is smooth across the ionization region. For efficient convection, then, it may be expected that, qualitatively, the nonadiabatic eigenfunctions will behave similarly to the adiabtic ones.

Also in Fig. 1 is shown the maximum lagrangean density variation obtained from nonlinear calculations (the density variation is scaled in such a way that the surface amplitude of relative radius variation is unity). As it is seen the linear $\Delta \rho / \rho$ for $\alpha \sim 1-2$ qualitatively resembles the nonlinear variation, what indicates that the effect of nonlinearity can be to a certain degree modeled by the inclusion of convection. Thus amplitude equations 


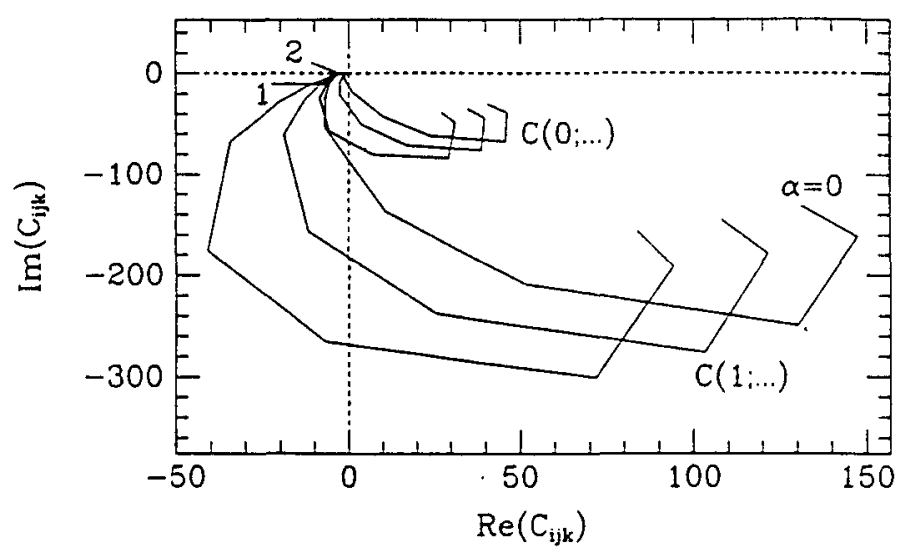

Fig. 2. Dependence of $C$ on $a$ for a model with $M=6 M_{\odot}, L=2280 L_{\odot}, \log \left(T_{\text {eff }}\right)=3.767$.

calculated basing on the results of linear pulsations with $\alpha \sim 1.5$ may better represent the behavior of nonlinear models.

\section{The Effect of Convection on Coupling in Cepheids}

To examine qualitatively the effect of convection on the strength of coupling the nonadiabatic formalism of TYI is adopted. This formalism, although it contains several simplifying assumptions, is used in the present case to obtain qualitative estimate of the coupling coefficients because (as it uses the second law of thermodynamics in phase of energy equation) it does not require the explicit specification of the mode of energy transport (radiative vs. radiative/convective).

In the TYI formalism the (second order nonresonant) coupling coefficients are calculated from $C_{i j k}=Q_{i j k} /\left(\sigma_{i}^{2} E K_{i}\right)$, where $E K_{i}$ and $\sigma_{i}$ are the kinetic energy and nondimensional frequency of mode $i$, respectively, and the integral $Q_{i j k}$ gives the strength of coupling between the three modes $\{i, j, k\}$ (TYI, Eq. (26)). The main contribution to this integral comes from the ionization region. With the increase of $\alpha$ the contribution from this region diminishes.

The dependence of $C$ on $\alpha$ is shown in Fig. 2. From this figure it is seen that in radiative models the coupling is strong and strongly nonadiabatic, $|\operatorname{Re} C| \sim|\operatorname{Im} C| \sim 10^{2}$. For $\alpha=1$ the coupling coefficients decrease to $|C| \sim 10$, and for larger $\alpha$ they become of comparable order to those calculated from adiabatic eigenfunctions, though they remain weakly nonadiabatic $(|\operatorname{Re} C| \gg|\operatorname{Im} C|>0)$.

For Cepheid models the coupling coefficients scale with model parameters approximately as $|C| \propto \lambda^{2}$, with $\lambda=\left(L / L_{\odot}\right)\left(M_{\odot} / M\right)^{2}$ (the fit is based on 
calculations for Cepheid models listed in Tables Ia,b in TYI). Hence with an increase of $L / M$, or a decrease of $M$ they increase rapidly.

\section{Conclusions}

The inclusion of convection in the background models reduces the steep gradients in the ionization region and hence results in a decrease of the amplitudes of thermodynamic eigenfunctions in this region. The coupling which is determined by the behavior of eigenfunction in the ionization region is thus reduced.

For Cepheid models the investigations of the effect of the strength of coupling on the occurrence of double mode behavior (Dziembowski and Kovács, 1984, Kovács and Kollath, 1988, Tanaka et al., 1991) have shown that the type of behavior is determined by the degree of nonadiabaticity and by the magnitude of coupling. Therefore in these stars the influence of convection should be included in the $a b$ initio calculation of nonadiabatic coupling coefficients.

For higher $L / M$ supergiants the convection becomes inefficient, so that it can not reduce the amplitudes of linear perturbations in the ionization region. Hence the coupling coefficients obtained from linear eigenvectors may lead to a significant overestimate of the strength of coupling. It would be interesting to see whether in extreme supergiant stars the approach of BK leads to amplitude equations which are compatible with the hydrodynamical calculations.

\section{References}

Buchler, J. R. and Goupil, M.: 1984, Astrophysical Journal 279, 394, BG.

Buchler J. R. and Kovács, G.: 1987, Astrophysical Journal 318, 232, BK.

Buchler, J. R., Moskalik, P. and Kovács, G.: 1991, Astrophysical Journal 380, 185.

Dziembowski, W.: 1977, Acta Astron. 27, 95.

Dziembowski, W. and Kovács, G.: 1984, Monthly Notices of the RAS 206, 497.

Kovács, G. and Buchler, J. R., 1989, Astrophysical Journal 346, 898.

Kovács, G. and Kolláth, Z.: 1988, in Multimode Stellar Pulsations, eds. G. Kovács, L. Szabados, and B. Szeidl (Kultura, Budapest), p. 33

Paczynski, B.: 1969, Acta Astron. 19, 1.

Pesnell, D. W. and Buchler, J. R.: 1986, Astrophysical Journal 303, 740.

Stellingwerf, R. F.: 1990, The Numerical Modelling of Nonlinear Stellar Pulsations, ed. by

J. R. Buchler, NATO ASI Series, vol. 302 (Kluwer Academic Publishers, The Netherlands), p.27.

Takeuti, M., Yamakawa, F. and Ishida, T.: 1991, Publications of the ASJ, submitted.

Tanaka, Y., Seya, K. and Takeuti, M.: 1991, Publications of the ASJ, submitted.

Unno, W., Osaki, Y., Ando, H., Saio, H. and Shibahashi, H.: 1989, Nonradial Oscilations of Stars, 2nd ed. (University of Tokyo Press, Tokyo). 